\title{
Presencia de Mimosa regnellil (Mimosoideae, Leguminosae) en Argentina
}

\author{
MATÍAS MORALES $1,2,3$
}

\begin{abstract}
Resumen: En el presente trabajo se confirma formalmente la presencia de Mimosa regnellii y se cita por primera vez a M. regnellii var. exuta para la Flora Argentina. Esta especie y su variedad se distribuyen en el extremo oriental de la provincia de Misiones, cerca de la frontera con Brasil. Se incluyen observaciones taxonómicas, geográficas y ecológicas. Con estos nuevos registros, el número de especies para la Flora de Argentina se eleva a 55 y el número de variedades a 25.
\end{abstract}

Palabras clave: Ecorregión de los Bosques Húmedos de Araucaria, Flora Argentina, Mimosa, Misiones, Mimosoideae, Myriophyllae.

\begin{abstract}
Summary: Presence of Mimosa regnellii (Mimosoideae, Leguminosae) in Argentina. In the present work, the presence of Mimosa regnellii is formally confirmed and $M$. regnellii var. exuta is reported for the first time in the Argentine Flora. This species and its variety occur in the eastern extreme of the Misiones province, near the Brazilian border. Taxonomic, geographic and ecological observations are included. The number of taxa of Mimosa in the Argentine Flora increases to 55 species and 25 varieties with these new reports.
\end{abstract}

Key words: Araucaria Moist Forest ecoregion, Argentine Flora, Mimosa, Misiones, Mimosoideae, Myriophyllae.

\section{INTRODUCCIÓN}

El género Mimosa está constituido por más de 530 especies (Simon et al., 2011), tiene distribución pantropical y subpantropical, $\mathrm{y}$ es predominantemente americano. Posee dos centros de diversificación, uno de ellos en el S de Sudamérica: Planalto de Brasil, al S de la Cuenca del Amazonas y las áreas adyacentes en el NE de Argentina, Paraguay y Uruguay, y el segundo en México, con centros minoritarios en Cuba, La Hispaniola, Cuenca del Orinoco y Madagascar. (Barneby, 1991).

\footnotetext{
1 Instituto de Recursos Biológicos, CIRN-CNIA, INTA. N. Repetto \& Los Reseros s.n., Hurlingham (1686), provincia de Buenos Aires, Argentina. E-mails: morales. matias@inta.gob.ar, mmorales0007@gmail.com

2 Facultad de Agronomía y Ciencias Agroalimentarias, Universidad de Morón. Cabildo 134, Morón (B1708JPD), pcia. de Buenos Aires, Argentina.

3 Consejo Nacional de Investigaciones Científicas y Técnicas (CONICET), Argentina.
}

Este género ha sido dividido en cinco secciones: Mimadenia Barneby, Batocaulon DC., Habbasia DC., Mimosa y Calothamnos Barneby (Barneby, 1991). Las secciones Mimosa y Calothamnos, que presentan flores haplostémonas, son consideradas las más evolucionadas (Simon et al., 2011; Bessega \& Fortunato, 2011).

La Argentina posee ca. 63 taxones del género Mimosa, entre los que se incluyen 54 especies (Fortunato et al., 2008). Éstas se hallan principalmente concentradas en la región mesopotámica, en las provincias de Misiones y Corrientes (Burkart, 1948), donde se localizan ca. 51 taxones, que constituyen el $81 \%$ de las mimosas de Argentina (Fortunato et al., 2008).

La sección Mimosa es una de las más evolucionadas del género y a su vez fue dividida en tres series, Myriophyllae Benth., Modestae Benth., y Mimosa. Los miembros de la serie Myriophyllae se caracterizan por su hábito arbóreo o arbustivo (raramente subarbustivo de porte erguido) y la presencia de pinnas pluriyugas con raquis no articulado, inflorescencias exertas, flores 
haplostémonas tetrámeras, con cáliz mayormente paleáceo-papiforme y fruto generalmente legumbre con valvas enteras o parcialmente enteras, caracteres que en su conjunto definen su identidad taxonómica dentro del género (Barneby, 1991).

La serie Myriophyllae es endémica de la región paranaense y comprende diez especies localizadas en su mayoría en el $\mathrm{S}$ de Brasil (desde Minas Gerais hasta Rio Grande do Sul), Paraguay y NE de Argentina, a excepción de una especie aislada en Serra dos Orgãos (Rio de Janeiro, Brasil) (Burkart, 1947; Barneby, 1991).

M. regnellii Benth., perteneciente a la serie Myriophyllae, es una especie frecuente en el $\mathrm{S}$ de Brasil, desde Minas Gerais hasta Santa Catarina (Burkart, 1979; Barneby, 1991; Dutra \& Morim, 2015), que exhibe elevada diversidad morfológica intraespecífica, especialmente en indumento, acúleos caulinares y fórmula foliar. Estos caracteres han permitido identificar cuatro variedades: $M$. regnellii var. regnellii, $M$. regnellii var. exuta Barneby, M. regnellii var. supersetosa (Burkart) Barneby y M. regnellii var. grossiseta Barneby (Barneby, 1991).

Como parte de la revisión que actualmente se realiza para la Flora Argentina, se confirma formalmente la presencia de $M$. regnellii en Argentina y se cita $M$. regnellii var. exuta en la provincia de Misiones. Se adjuntan descripciones de la especie y la variedad, su distribución geográfica y ecología. Se incluye una clave para identificar a los representantes de la serie Myriophyllae en Argentina.

\section{Materiales y Métodos}

Se revisaron especímenes del género Mimosa depositados en BA, BAB, CTES, MBM, MO, NY, SI, los cuales fueron visitados desde 2005 hasta la fecha. Los especímenes fueron caracterizados morfológicamente e identificados de acuerdo con la propuesta de Barneby (1991).

\section{Tratamiento taxonómico}

\section{Ser. Myriophyllae Benth.}

Arbustos arborescentes a pequeños árboles, raro subarbustos erguidos. Indumento híspido-estrigoso. Tallos generalmente inermes. Hojas con pinnas 2-24-yugas; foliólulos 15-80-yugos por pinna, 1,5-11 × 0,5-3 mm, 1-3-nervados, con margen setoso discontinuo y venación secundaria poco visible. Inflorescencias exertas, pseudoracemosas, constituidas por cabezuelas esferoides o raramente espigas. Flores haplostémonas, tetrámeras, tetrandras, con cáliz paleáceo papiforme o más raramente campanulado. Fruto craspedio o legumbre de valvas enteras por aborto de las septas.

\section{Clave para la diferenciación de las especies de Mimosa Sección Mimosa Serie Myriophyllae en la Argentina}

1. Estilo menor de $2 \mathrm{~mm}$, menos de la mitad del largo de los estambres. Filamentos estaminales rojizos. Anteras de 0,7-0,9 mm de largo........................................ M. myriophylla

1'. Estilo de $6 \mathrm{~mm}$ de largo o mayor, igualando o superando el largo de los estambres. Filamentos estaminales rosados o liláceos. Anteras de 0,3$0,6 \mathrm{~mm}$ de largo............. regnellii (var. exuta)

Mimosa regnellii Benth. Linnaea 22: 529. 1849. Tipo: Brasil. Minas Gerais, Habitat ad Caldas prov. Minas Geraës, Regnell coll. II n. 93 (Holotipo K $=\mathrm{NY}$ Neg. 1837; Isotipo $\mathrm{S}=\mathrm{NY}$ Neg. 11844, P! (visto en imagen digital), US). Fig. 1.

Árboles y arbustos arborescentes de hasta 3-4 $\mathrm{m}$ de altura. Tallos inermes, híspidos a estrigosos. Hojas con estípulas 3-10 × 0,5-2 mm, pubescentes, persistentes; eje principal (raquis primario y pecíolo) de $2-15 \mathrm{~cm}$ de largo, incluyendo pecíolo de 0,5-4,5 $\mathrm{cm}$ de largo; pinnas 4-13yugas; foliólulos 15-36-yugos por pinna, 3-7 $\times$ 1-2 mm, estrechamente lanceolados a ovados, a veces oblicuos, base semicordada, ápice agudo a mucronado, glabros a pubescentes en ambas caras, con margen generalmente ciliado, 1-4-nervados, venación secundaria inmersa en la lámina o poco visible. Inflorescencias exertas, en pseudoracimos de cabezuelas, de hasta $40 \mathrm{~cm}$ de largo, con pedúnculos de 4-16 mm de largo y cabezuelas 5-8 $\mathrm{mm}$ de diámetro, esferoides; brácteas 1,5-3,5 × 0,5 $\mathrm{mm}$, oblanceoladas. Flores tetrámeras, tetrandras, 


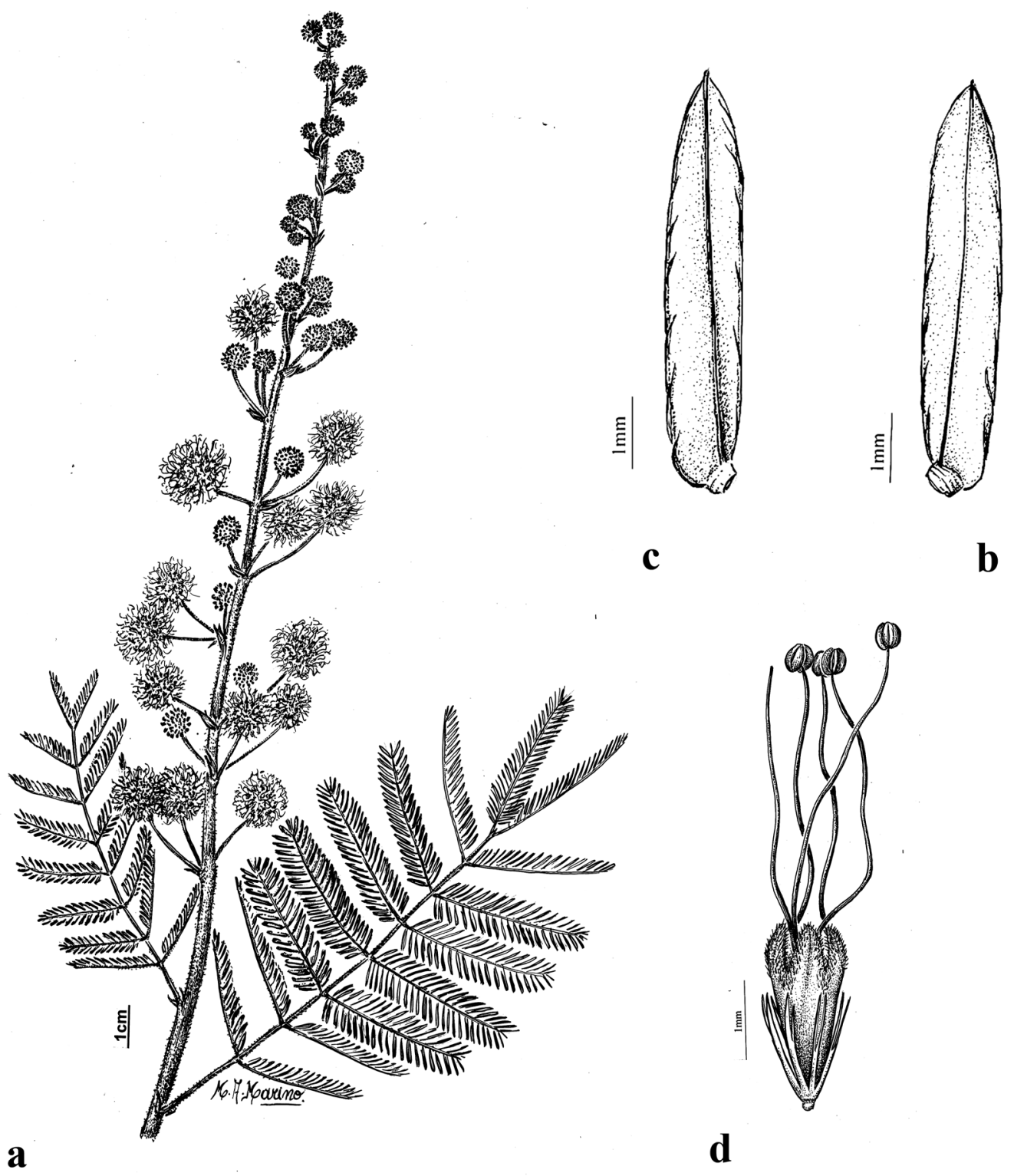

Fig. 1. Mimosa regnellii Benth. var. exuta Barneby. a. Rama con flores. b. Foliólulo, haz. c. Foliólulo, envés. d. Flor. Dibujado del espécimen A. L. Cabrera \& A. A. Sáenz 29233 (SI).

con cáliz 1,5-3 mm de largo, paléaceo-papiforme; corola 2,5-3,5 $\mathrm{mm}$ de largo, pubérula; androceo con filamentos estaminales libres, rosados. Fruto generalmente legumbre con valvas enteras por aborto de las septas del fruto 4,5-10 × 3,5-7 mm, híspidos en replum y valvas. Semillas generalmente 1-2 por fruto, $2-3 \times 2-3 \mathrm{~mm}$, obovoides, castañas.
Observaciones taxonómicas. $M$. regnellii se puede incluir sin problemas en Mimosa serie Myriophyllae, dado su hábito arbóreo/arbustivo, hojas con pinnas y foliólulos pluriyugos, la presencia de flores tetrámeras, tetrandras, con cáliz paleáceopapiforme y agrupadas en cabezuelas esferoides, las cuales forman pseudoracimos exertos. 
El hábito arbóreo/arbustivo de $M$. regnellii le permite diferenciarse de las especies de Myriophyllae que son subarbustos o sufrútices de campos, como M. candelabrum Hassl. y $M$. altoparanensis Burkart, dos especies endémicas de Paraguay.

De acuerdo con Barneby (1991), el nervio medio de los foliólulos de $M$. regnellii es excéntrico y divide al foliólulo en una relación 1:2. Este carácter permitiría distinguir esta especie de $M$. micropteris Benth. y M. deceptrix Burkart, que tienen venación simétrica, ya que el nervio medio divide el foliólulo en similares proporciones. $M$. deceptrix es también diferente de $M$. regnellii por las inflorescencias y la fórmula foliar: $M$. deceptrix posee hojas mucho más amplias e inflorescencias más largas [eje primario de 7-16 cm de largo; pinnas 9-16-yugas, folíolos 2546-yugos y pseudoracimos de 50-80 $\mathrm{cm}$ de largo].

Las inflorescencias primarias -cabezuelas esferoides con brácteas que apenas sobresalen del conjunto de flores- son caracteres que distinguen la especie en estudio de $M$. balduinii Burkart, un endemismo del S de Brasil (Rio Grande do Sul) que posee flores dispuestas en espigas, y de $M$. chartostegia Barneby, endémica del estado brasileño de Paraná, la cual posee inflorescencias con brácteas florales notablemente mayores que las de las inflorescencias en cabezuelas (Barneby, 1991).

La fórmula foliar y el tipo de cáliz permiten diferenciar adecuadamente a $M$. regnellii [pinnas 4-13-yugas, foliólulos 15-36-yugos y cáliz paleáceo- papiforme] de $M$. glazioui Benth. [pinnas 2-3-yugas, foliólulos 16-22-yugos y cáliz campanulado].

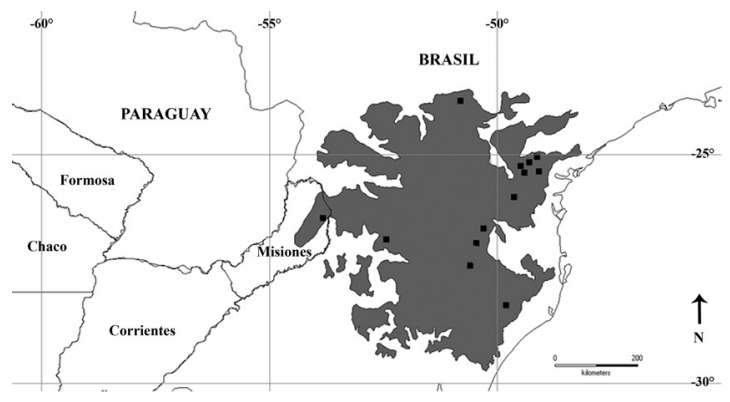

Fig. 2. Distribución geográfica de Mimosa regnellii var. exuta. En gris, el área de la ecorregión Selva de Pino Paraná.
M. regnellii forma parte del grupo de especies de Myriophyllae con estilo comparativamente largo (de $6 \mathrm{~mm}$ o más), filamentos estaminales lilas a rosados y anteras de hasta 0,6 mm de largo. Estos caracteres la diferencian claramente de M. myriophylla Bong. ex Benth. y M. callidryas Barneby, cuyos estilos son menores que $M$. regnellii [menores a $2 \mathrm{~mm}$ de largo] y la mitad del largo de los filamentos estaminales rojos, por lo que son levemente exertos; asimismo, las anteras en estas dos especies son mayores [0,7-0,9 $\mathrm{mm}$ de largo] que las de M. regnellii.

Distribución geográfica. M. regnellii posee una distribución discontinua predominantemente en el $\mathrm{S}$ de Brasil, crece en algunas localidades aisladas de Minas Gerais y luego aparece con una amplia distribución desde São Paulo hasta Santa Catarina. En la revisión que se está llevando a cabo sobre las especies del género Mimosa en Argentina, se confirmó la presencia de $M$. regnellii en este país. Esta especie ha sido coleccionada en las inmediaciones de Dos Hermanas, localidad cercana a Bernardo de Irigoyen, próximo a la frontera con Brasil (Fig. 2).

Burkart (1948) no incluyó a M. regnellii dentro de su tratamiento de las especies argentinas del género, aunque sugirió que debe incorporarse al catálogo de las especies nativas. La exclusión de $M$. regnellii podría explicarse por la falta de especímenes de referencia, pues su cita se basó en un solo espécimen, Niederlein 1117 (BA). El mismo corresponde efectivamente a $M$. regnellii, pero la localidad citada, Boa Vista, parece dudosa por su etimología y antigüedad de la colección. Según Burkart (1948) Boa Vista sería un cerro entre Fracrán y San Pedro, en el centro de Misiones; este dato no he podido corroborarlo hasta el momento, aunque su localización coincide con el ambiente donde puede aparecer la especie en Argentina.

Posteriormente, Barneby (1991) y Fortunato et al. (2008) no consideraron a $M$. regnellii como nativa de Argentina. Sin embargo, a través de la revisión de los herbarios mencionados he podido encontrar colecciones relativamente recientes que permiten formalmente confirmar la presencia de la especie en el país.

De acuerdo con la información actualizada de la Flora del Cono Sur (Fortunato et al., 2008) y la presente contribución, el número de especies de 
Mimosa para la Flora Argentina se eleva a 55, el número de variedades a $25 \mathrm{y}$ el número de taxones específicos e infraespecíficos a 64 , incluyendo hasta el momento dos especies pertenecientes a la sección Mimosa serie Myriophyllae.

Ecología. El complejo M. regnellii presenta su máxima diversificación en los bosques de Araucaria angustifolia (Bertol.) Kuntze o bosques paranaenses (especialmente en bordes de bosque y áreas disturbadas) y campos (campo limpo, campo de altitude) del S de Brasil (Burkart, 1979; Dutra \& Morim, 2015). Estos ecosistemas están incluidos en la ecorregión denominada Selva de Pino Paraná o Bosques Húmedos de Araucaria (Olson et al., 2001). Es interesante observar que el patrón de distribución de $M$. regnellii coincide ampliamente con el espacio que ocupa dicha ecorregión en Brasil y Argentina. En éste último país $M$. regnellii se encontró en las áreas que aún conservan importantes relictos de A. angustifolia, en el E de la provincia de Misiones, en sus áreas más elevadas (Dos Hermanas, donde se halló $M$. regnellii, es la localidad de mayor altitud de la provincia, con $700 \mathrm{~m} \mathrm{~s}$. n. m.).

Fenología. M. regnellii florece desde septiembre hasta febrero, y de acuerdo con Burkart (1979) fructifica a partir de marzo. Los frutos pueden persistir en la planta varios meses luego de su dehiscencia.

Mimosa regnellii var. exuta Barneby. Mem. New York Bot. Gard. 65: 546. 1991. Tipo: Brasil. Paraná, Rio Branco do Sul, Itaperucu, ad marginem silvulae, 29-XI-1912, Dusen 13869 (Holotipo S = NY Neg. 11846!). Fig. 1.

Hojas con eje principal de 6-15 cm de largo; pecíolo de 1,5-4,5 cm de largo; foliólulos de hasta 4-7 × 1-1,8 mm, generalmente lanceoladooblongos, glabros en una o ambas caras, relación largo: ancho $3: 1-4,7: 1$.

Especimenes selectos examinados. BRASIL. Edo. Paraná: Mun. Almirante Tamandaré, Marmeleiro, 30-I-1996, O. S. Ribas et al. 1071 (NY); Mun. Balsa Nova, Cristo, 18-IV-2009, R. H. Fortunato et al. 9496 (BAB); Mun. General Carneiro, Jangada, 05-XII-1989, G. G. Hatschbach
\& V. Nicolack 53562 (NY); Mun. Palmeira, Fazenda Santa Rita, 21-I-1982, P. I. Oliveira 300 (NY); Mun. Quatro Barras, on roadside, 07-IV1958, L. Williams 18782 (NY); Mun. São Jerónimo da Serra, Rio de Tigre, 24-II-1957, G. Hatschbach 3596 (SI); Mun. Tunas do Paraná, Parque Estadual de Campinhos, 19-II-1999, J. M. Silva et al. 2878 (NY). Edo. Santa Catarina: Mun. Curitibanos, Rod. BR-470, km 203, 11-II-1996, O. S. Ribas et al. 1256 (MBM); Mun. Faxinal dos Guedes, dry bank by road to Xanxerê, 08-XI-1964, L. B. Smith \& R. M. Klein 13060 (SI); Mun. Mafra, campo 7 km NW of Mafra on the road to Barracas (20 km), 13-III1957, Smith \& Klein 12100 (SI). ARGENTINA. Prov. Misiones: Dpto. General Belgrano, Dos Hermanas, a $13 \mathrm{~km}$ de Bernardo de Yrigoyen, 16II-1978, A. L. Cabrera \& A. A. Sáenz 29233 (SI); de Piñalito a Dos Hermanas, Ruta Nacional 14, a 6 $\mathrm{km}$ del asfalto a Yrigoyen, 16-II-1996, O. Morrone et al. 913 (CTES282313).

Observaciones taxonómicas. En M. regnellii, y especialmente en la var. exuta, se ha observado que el nervio medio no es estrictamente excéntrico en todo su recorrido, como lo había descripto previamente Barneby (1991) indicando que es un carácter diferencial de la especie. En la variedad exuta los foliólulos son angostos, lanceoladooblongos y estrechos, a veces oblicuos y debido a ello, el nervio medio suele ser más excéntrico en su parte inferior. Por lo tanto, la identificación de este taxón infraespecífico podría ser dificultosa (Barneby, 1991) si no se tiene en cuenta este detalle.

La variedad exuta difiere del resto de las variedades de $M$. regnellii por caracteres ligados a la fórmula foliar, indumento y forma y pubescencia de los foliólulos. M. regnellii var. exuta y M. regnellii var. regnellii difieren entre sí por la diferente pubescencia de los foliólulos (Barneby, 1991). M. regnellii var. exuta posee foliólulos glabros o apenas pubescentes en el envés, mientras la variedad típica posee los foliólulos densamente pubescentes en ambas caras.

Por otro lado, la variedad exuta posee hojas mucho más amplias y foliólulos de diferente morfología que las variedades supersetosa y grossiseta [eje principal de las hojas $2-6 \mathrm{~cm}$ de largo; pecíolos 1-1,5 cm de largo, y foliólulos generalmente semi-ovados a ovados]. Además, el indumento en la var. exuta está formado por setas 
angostas en su base [0,1-0,2 $\mathrm{mm}$ de diámetro en su base], mientras que la var. grossiseta posee un indumento con setas basalmente dilatadas [mayores a $0,3 \mathrm{~mm}$ de diámetro en la base].

Las diferencias entre las variedades de $M$. regnellii, propuestas por Barneby (1991), están siendo detalladamente analizadas (Matías Morales, datos no publicados) debido a la presencia de formas intermedias, en especial en cuanto a las dimensiones de las hojas y la pubescencia foliar.

Distribución geográfica. M. regnellii var. exuta se encuentra distribuida en el S de Brasil, en Paraná y Santa Catarina, así como en las áreas adyacentes de la provincia de Misiones (Argentina) (Fig. 2), lo que descartaría que sea un endemismo de Brasil (Barneby, 1991; Dutra \& Morim, 2015). Esta variedad convive en simpatría en gran parte de su área de distribución con otras variedades de $M$. regnellii, como $M$. regnellii var. supersetosa y $M$. regnellii var. grossiseta. A diferencia de estas tres variedades, la variedad típica de $M$. regnellii sólo es hallada en Minas Gerais, en el extremo norte de distribución de la especie (Barneby, 1991).

Ecología. Esta variedad crece en los bosques de Araucaria y campos del S de Brasil. De acuerdo con mis observaciones de campo y la bibliografía (Burkart, 1979; Barneby, 1991) es heliófita y suele ser encontrada en bordes de bosques y de caminos, así como en áreas abiertas o deforestadas (capoeiras) con vegetación secundaria.

\section{Agradecimientos}

Agradezco a Renée Fortunato, por haberme guiado en el camino de la botánica desde mis inicios. Dejo mis reconocimientos a los curadores de los herbarios visitados por facilitar el acceso a las colecciones estudiadas y a Angélica Marino por las ilustraciones de la especie. Los viajes del autor a diferentes herbarios nacionales y extranjeros han sido financiados por INTA, Troels Myndel Foundation (convocatorias 2009 y 2010), Universidad de Morón (PID B.06-001/06, 06-001/08 y 06-005/12), Agencia Nacional de Promoción Científica y Tecnológica (PICT 08212011) y CONICET (convocatoria 2011 para estadías cortas en el exterior).

\section{Bibliografía}

BARNEBY, R. C. 1991. Sensitivae Censitae: A description of the genus Mimosa Linnaeus (Mimosaceae) in the New World. Mem. New York Bot. Gard. 65: 1-835.

BESSEGA, C. \& R. H. FORTUNATO. 2011. Section Mimadenia: its phylogenetic relationships within the genus Mimosa (Leguminosae, Mimosoideae) using plastid trnL-F sequence data. Austr. Syst. Bot. 24: 104-110.

BURKART, A. 1947. Leguminosas nuevas o críticas II. Darwiniana 7: 504-540.

BURKART, A. 1948. Las especies de Mimosa de la Flora Argentina. Darwiniana 8: 9-231.

BURKART, A. 1979. Mimosa. En: REITZ, P. R. (ed.) Flora Ilustrada Catarinense: Leguminosas Mimosoideas (1): 128-257. Herbário Barbosa Rodrigues, Itajaí.

DUTRA, V. F. \& M. P. MORIM. 2015. Mimosa. En: Lista de Espécies da Flora do Brasil. Jardim Botânico do Rio de Janeiro. Disponible en: <http:// floradobrasil. jbrj.gov.br/jabot/floradobrasil/ FB23084>. [Consultado el 15 de Julio de 2015].

FORTUNATO, R. H., R. BEYHAUT, R. L. COSTA BORTOLUZZI, E. V. GÓMEZ SOSA, P. IZAGUIRRE, H. C. LIMA, S. T. S. MIOTTO, L. R. MOURA BAPTISTA \& E. A. ULIBARRI. 2008. Fabaceae. En: ZULOAGA, F., O. MORRONE \& M. J. BELGRANO (eds.), Catálogo de Plantas Vasculares del Cono Sur 2: 2078-2251. Actualizado en: ZULOAGA, F. O. \& M. J. BELGRANO (eds.). 2015. Catálogo de las plantas vasculares del Cono Sur (Argentina, sur de Brasil, Chile, Paraguay y Uruguay). Disponible en: $<$ http://www.darwin.edu. ar/Proyectos/FloraArgentina/fa.htm $>$ [consultado el 15 de julio de 2015].

OLSON, D. M., E. DINERSTEIN, E. D. WIKRAMANAKAYE, N. D. BURGESS, G. V. N. POWELL, E. C. UNDERWOOD, J. A. D'AMICO, I. ITOUA, H. E. STRAND, J. C. MORRISON, C. J. LOUCKS, T. F. ALLNUTT, T. H. RICKETTS, Y. KURA, J. F. LAMOREUX, W. W. WETTENGEL, P. HEDAO \& K. R. KASSEM. 2001. Terrestrial ecoregions of the World: a new map of life on earth. Bioscience 51: 933-938.

SIMON, M. F., R. GRETHER, L. P. DE QUEIROZ, T. E. SÄRKINEN, V. F. DUTRA \& C. E. HUGHES. 2011. The evolutionary history of Mimosa (Leguminosae): toward a phylogeny of the sensitive plants. Amer. $J$. Bot. 98: 1201-1221.

Recibido el 25 de febrero de 2015, aceptado el 21 de mayo de 2015. 